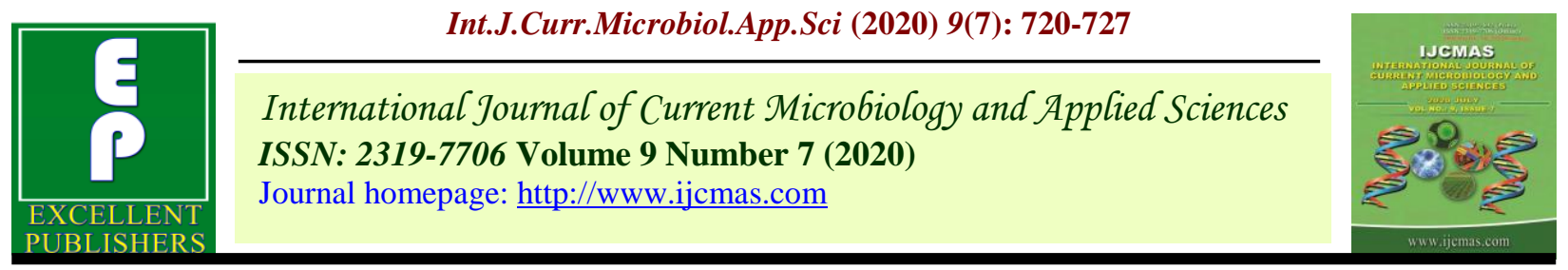

Original Research Article

https://doi.org/10.20546/ijcmas.2020.907.083

\title{
Combining Ability and Heterosis for Seed Yield and its Attributing Traits in Indian Mustard (Brassica juncea L. Czern \& Coss)
}

\author{
Ashish Sahu*, J. L. Salam, Surbhi Verma and Stanley Samuel
}

Department of Genetics and Plant Breeding, S.G. College of Agriculture and Research Station, Kumhrawand, Jagdalpur, Indira Gandhi Krishi Vishwavidyalaya, Raipur, (Chhattisgarh), India

*Corresponding author

\section{A B S T R A C T}

Keywords

Grain yield, SCA

value, heterosis

effect

Article Info

Accepted:

08 June 2020

Available Online:

10 July 2020
Combining ability analysis was done for nine parents including five female lines and four testers male with their twenty crosses. Laxmi and RH - 406 was conferred as the great general combiner distinguished for "grain yield per plant" because of its positive GCA impact. Among the four testers, Varuna, PM - 27 and Pusa Bold were distinguished as great combiner for the same trait and other related characteristics. "Lxmi" was recognized as great general combiner for the greater part of the yield related attributes. C.G.Sarson $x$ Pusa showed the highest SCA value followed by Pusa Agrime x Varuna for "grain yield per plant". Among the hybrids C.G. Sarson $\mathrm{x}$ Kranti and Laxmi x Varuna were reported to be the best for heterosis effect for all characters taken under study.

\section{Introduction}

Among the oilseed crops mustard rank second in world with an area of 37.0 mha with a production of $18-50 \mathrm{q}$ per ha. In India the area, production and productivity are $6.3 \mathrm{mha}$, $7.6 \mathrm{~m}$ tones and $11.90 \mathrm{q}$ per ha respectively (Anonymous 2016). Mustard ranks second in area after ground nut and third in production after soybean (Nirajan et al., 2014). Uttar Pradesh, Rajasthan, Madhya Pradesh, Punjab, Haryana, West Bengal, Assam and Bihar are major mustard growing state in India. In compared to other species of Brassica the crop mustard the crop mustard has higher adaptability in accordance with tolerant to biotic and abiotic stresses (Yadava et.al., 2010).

In plant breeding programme, it is essential to select parents with desirable characteristics with good general combining ability (GCA) effects for yield and high estimates of specific combining ability (SCA) effects of hybrids. 
A very powerful tool to estimate combining ability effects is Line $\mathrm{x}$ Tester analysis introduced by Kempthorne (1957). It imparts information on GCA and SCA effects of parents. It also helps in estimation of various types of gene actions governing the inheritance of characters and thereby, helps to select parents and crosses which are more desirable and superior in performance.

\section{Materials and Methods}

Using five lines viz. Laxmi, Pusa Agrime, DRMR IJ - 31, C.G.Sarson, RH - 406 as female parents and four testers viz. Pusa Bold, Kranti, Varuna and PM - 27 as male parents, twenty crosses were produced through $\mathrm{L}$ x $\mathrm{T}$ mating design at "Research cum Instructional Farm of S.G. College of Agriculture and Research Station, Kumhrawand, Jagdalpur, Bastar. The crosses were attempted during rabi season 2017-18. The parents and their crosses were evaluated during rabi 2018-19. One checks namely NRCBH - 101 were also planted. maintaining aPlot size $-1.8 \times 5 \mathrm{~m}$, Spacing - $30 \times 10 \mathrm{~cm}$. Observations were recorded for twelve yield parameters "days to $50 \%$ flowering, days to maturity, plant height (cm), number of primary branches per plant, number of secondary branches per plant, length of siliqua $(\mathrm{cm})$, length of main raceme (cm),number of siliqua on main raceme, number of seed per siliqua, 1000 seed weight $(\mathrm{g})$, oil content $(\%)$ and harvest index $(\%)$."

\section{Results and Discussion}

The results of $\mathrm{L} x \mathrm{~T}$ analysis for assessment of combining ability are discussed under the following sub-heads:

\section{ANOVA for line $\mathrm{x}$ tester analysis}

Table 2 presenting ANOVA for Line $\mathrm{x}$ Tester Analysis shows highly significant was observed among treatments for all the characters under study. The differences were found to be highly significant also for both" parents and hybrids" for all the characters under study.

\section{Combining ability analysis for GCA and SCA effects}

Character wise results of combining ability analysis for GCA and SCA effects are briefly described below.

Among the lines, DRMR IJ - 31(-1.067) showed negative highly significant effect. Among the testers, Kranti (-0.033) and Varuna (-0. 33) showed negative highly significant GCA effects. The negative GCA effects indicate earliness.

Among the lines, Pusa Agrime (- 15.99) and DRMR IJ - 31 showed negative highly significant GCA effect. Among the testers, Pusa Bold showed negative highly significant GCA effects.

GCA effects for primary branches per plant are ranged from -0.13 (Kranti) to 0.33(Varuna). Among the line C.G.Sarson shows negative significant GCA effect where as highly positive GCA effect was observed in Laxmi and Pusa Agrime.

Among the tester Pusa Bold has negatively significant GCA effect whereas Varuna has highly positive GCA effect. Varuna, Laxmi and Pusa Bold were registered the good general combiners for number of primary branches per plant.

GCA effects for secondary branches per plant are ranged from -0.017 (Pusa Agrime) to $0.417(\mathrm{PM}$ - 27). Among the Parents C.G.Sarson, Kranti and Varuna shows negative significant GCA effect where as highly positive GCA effect was observed in Laxmi, PM - 27 and Pusa Bold. 
The parents PM - 27 (0.41), Pusa Bold (0.35) and Laxmi (0.31) were registered the good combiners for number of secondary branches per plant. Among the lines, Pusa Agrime (0.128 ) showed negative highly significant effect. Among the testers, Varuna (-0.130) showed negative highly significant GCA effects. None of the parents were recorded positive significant for the character length of siliqua.

Both line and tester were found to have highly significant GCA effect. The lines DRMR IJ31 and C.G. Sarson showed negative highly significant GCA effects where as highly positive GCA effect was observed in RH 406, Laxmi and Pusa Agrime. Among the tester Pusa Bold and Kranti has negatively significant GCA effect where as Varuna and PM -27 has highly positive GCA effect. The positive GCA effects indicate their usefulness in breeding for length of main raceme.

Both line and tester were found to have highly significant GCA effect. The lines DRMR IJ31, Pusa Agrime and C.G. Sarson showed negative highly significant GCA effects where as highly positive GCA effect was observed in $\mathrm{RH}-406$ and Laxmi.
Among the tester Kranti has negatively significant GCA effect whereas Varuna has highly positive GCA effect. The positive GCA effects indicate their usefulness in breeding for number of siliqua on main raceme. Both line and tester were found to have highly significant GCA effect. The lines C.G. Sarson showed negative significant GCA effects where as highly positive GCA effect was observed in Laxmi. Among the tester Kranti has negatively significant GCA effect whereas no positive GCA effects were reported. The positive GCA effects indicate their usefulness in breeding for number of seeds per siliqua.

Among the line, C.G. Sarson showed positive highly significant GCA effects. Among the testers, Varuna showed positive highly significant GCA effects. Positive effects of parents indicate their usefulness in breeding of developing best hybrids.

Both line and tester were found to have highly significant GCA effect. The lines C.G. Sarson and DRMR IJ - 31 showed negative significant GCA effects where as highly positive GCA effect was observed in Laxmi and pusa Agrime.

Table.1 Details of genotypes used in investigation

\begin{tabular}{|l|l|}
\hline Genotypes & Source \\
\hline Female Parents & S.G.CARS, Jagdalpur (Bastar),India \\
\hline Pusa Agrime & IARI, New Delhi, India \\
\hline $\begin{array}{l}\text { C.G.Sarson } \\
\text { RH - 406 }\end{array}$ & $\begin{array}{l}\text { S.G.CARS, Jagdalpur (Bastar),India } \\
\text { S.G.CARS, Jagdalpur (Bastar),India }\end{array}$ \\
\hline DRMR IJ - 31 & S.G.CARS, Jagdalpur (Bastar),India \\
\hline Male Parents & IARI, New Delhi, India \\
\hline Pusa Bold & S.G.CARS, Jagdalpur (Bastar),India \\
\hline Kranti & S.G.CARS, Jagdalpur (Bastar),India \\
\hline Varuna & S.G.CARS, Jagdalpur (Bastar),India \\
\hline PM - 27 & IGKV, Raipur (C.G.), India \\
\hline Checks & \\
\hline NRCBH - 101 & \\
\hline
\end{tabular}


Table.2 Analysis of Variance for L X T

\begin{tabular}{|c|c|c|c|c|c|c|c|c|}
\hline \multirow[t]{4}{*}{ Source of variation } & \multirow[t]{4}{*}{ df } & \multicolumn{6}{|c|}{ Mean Squares } & \multirow[b]{2}{*}{ Harvest } \\
\hline & & Length of & Number of & Number of & 1000 seed & Oil & Seed yield & \\
\hline & & Main & Siliqua on & Seed/siiqua & Weight & $\begin{array}{l}\text { Content } \\
(\%)\end{array}$ & Per plant & Index $(\%)$ \\
\hline & & Raceme $(\mathrm{cm})$ & Main raceme & & (g) & & (g) & \\
\hline Replication & 2 & 4.38 & $16.63 * *$ & $5.41 * *$ & $0.11 *$ & 0.79 & 0.58 & 5.26 \\
\hline Treatments & 28 & $142.75^{* *}$ & $45.97 * *$ & $2.55 * *$ & 0.04 & $4.46 * *$ & $7.21 * *$ & $10.61 * *$ \\
\hline Parents & 8 & $82.66 * *$ & $38.34 * *$ & $3.00 * *$ & 0.02 & $3.73 * *$ & $3.94 * *$ & $9.02 * *$ \\
\hline Parents(Lines) & 4 & $63.67 * *$ & $22.06 * *$ & $2.26 * *$ & 0.01 & $4.00 * *$ & $3.90 * *$ & $9.59 * *$ \\
\hline Parents(Testers) & 3 & $129.72 * *$ & $72.11 * *$ & $1.22 * *$ & 0.02 & 1.62 & $2.72 *$ & $8.53 * *$ \\
\hline $\operatorname{Parents}(\mathrm{L}$ v/s T) & 1 & $17.46^{* *}$ & 2.14 & $11.26^{* *}$ & 0.04 & $9.04 * *$ & $7.82 * *$ & 6.76 \\
\hline Parents v/s Crosses & 19 & 0.81 & $26.89 * *$ & $5.52 * *$ & 0.05 & 0.059 & $67.50 * *$ & $52.63 * *$ \\
\hline Crosses & 1 & $175.52 * *$ & $50.19 * *$ & $2.21 * *$ & 0.05 & $5.00 * *$ & $5.41 * *$ & $9.07 * *$ \\
\hline Line Effect & 4 & 227.30 & 41.72 & 2.183 & 0.08 & 4.03 & 9.00 & 11.08 \\
\hline Tester Effect & 3 & 136.09 & 27.61 & 0.733 & 0.06 & 4.03 & 8.90 & 3.60 \\
\hline Line $*$ Tester Effect & 12 & $168.11^{* *}$ & $58.65^{* *}$ & $2.59 * *$ & 0.03 & $5.56 * *$ & $3.33 * *$ & $9.76^{* *}$ \\
\hline Error & 56 & 2.163 & 2.16 & 0.27 & 0.02 & 0.77 & 0.76 & 1.75 \\
\hline Total & 86 & 47.98 & 16.76 & 1.13 & 0.03 & 1.97 & 2.86 & 4.71 \\
\hline
\end{tabular}


Table.3.1 General combining ability effects of parents for different characters

\begin{tabular}{|c|c|c|c|c|c|c|}
\hline Characters & $\begin{array}{l}\text { Days to } \mathbf{5 0 \%} \\
\text { Flowering }\end{array}$ & $\begin{array}{l}\text { Days to } \\
\text { Maturity }(\mathrm{cm})\end{array}$ & $\begin{array}{l}\text { Plant } \\
\text { height }\end{array}$ & \multirow{2}{*}{$\begin{array}{l}\text { Number of } \\
\text { Primary } \\
\text { Branches/ plant }\end{array}$} & \multirow{2}{*}{$\begin{array}{l}\text { Number } \\
\text { Secondary } \\
\text { Branches } \\
\text { /plant }\end{array}$} & \multirow{2}{*}{$\begin{array}{l}\text { Length of } \\
\text { siliqua } \\
(\mathrm{cm})\end{array}$} \\
\hline & & & & & & \\
\hline Laxmi & 0.600 & -0.033 & 8.778 ** & $0.300 *$ & $0.317 *$ & 0.047 \\
\hline Pusa Agrim & 0.350 & -0.533 & $-15.997 * *$ & $0.300 *$ & -0.017 & $-0.128 *$ \\
\hline C.G. Sarson & $-1.067 * *$ & 0.217 & $-6.305 * *$ & -0.200 & 0.067 & -0.020 \\
\hline RH-406 & $0.767 *$ & -0.033 & $6.562 * *$ & $-0.617 * *$ & $-0.433 * *$ & 0.072 \\
\hline DRMR IJ - 31 & -0.650 & 0.383 & $6.962 * *$ & 0.217 & 0.067 & 0.030 \\
\hline Pusa Bold & -0.100 & -0.550 & $-6.180 * *$ & $-0.400 * *$ & $0.350 * *$ & 0.057 \\
\hline Kranti & -0.033 & 0.450 & 1.020 & -0.133 & $-0.383 * *$ & -0.023 \\
\hline Varuna & -0.300 & 0.117 & $1.893 * *$ & $0.333 * *$ & $-0.383 * *$ & $-0.130 *$ \\
\hline PM-27 & 0.433 & -0.017 & $3.267 * *$ & 0.200 & $0.417 * *$ & 0.097 \\
\hline
\end{tabular}

*Significant at $\mathrm{p}=0.05 \%$ level, $* *$ Significant at $\mathrm{p}=0.01 \%$ level

Table.3.2 General combining ability effects of parents for different characters

\begin{tabular}{|c|c|c|c|c|c|c|c|}
\hline \multirow{3}{*}{ Characters } & \multirow{3}{*}{$\begin{array}{l}\text { Length of } \\
\text { Main } \\
\text { Raceme }(\mathrm{cm})\end{array}$} & Number of & Number of & \multirow{3}{*}{$\begin{array}{l}1000 \text { seed } \\
\text { Weight } \\
(\mathrm{g})\end{array}$} & \multirow{3}{*}{$\begin{array}{c}\text { Oil } \\
\text { Content }(\%)\end{array}$} & \multirow{3}{*}{$\begin{array}{c}\text { Seed } \\
\text { Yield/ } \\
\text { Plant (g) }\end{array}$} & \multirow{3}{*}{$\begin{array}{c}\text { Harvest } \\
\text { Index }(\%)\end{array}$} \\
\hline & & Siliqua on & Seed/siiqua & & & & \\
\hline & & \multicolumn{2}{|l|}{ Main raceme } & & & & \\
\hline Laxmi & $3.075 * *$ & $2.017 * *$ & $0.350 *$ & $0.114 *$ & -0.390 & $1.079 * *$ & $1.290 * *$ \\
\hline Pusa Agrim & $1.725 * *$ & $-1.400 * *$ & 0.183 & -0.031 & 0.512 & $0.704 * *$ & -0.058 \\
\hline C.G. Sarson & $-2.367 * *$ & $-0.900 *$ & 0.017 & -0.005 & -0.269 & $-0.833 * *$ & -0.476 \\
\hline RH-406 & $-6.450 * *$ & $-1.733 * *$ & $-0.733 * *$ & 0.038 & $0.725 * *$ & $-0.787 * *$ & 0.492 \\
\hline DRMR IJ - 31 & $4.017 * *$ & $2.017 * *$ & 0.183 & $-0.117 *$ & $-0.578 *$ & -0.163 & $-1.24 * *$ \\
\hline Pusa Bold & $-0.843 *$ & -0.183 & 0.033 & -0.035 & 0.038 & -0.296 & 0.654 \\
\hline Kranti & $-3.903 * *$ & $-1.783 * *$ & $-0.300 *$ & -0.073 & -0.442 & $-0.728 * *$ & -0.190 \\
\hline Varuna & $2.430 * *$ & $1.417 * *$ & 0.033 & 0.068 & $0.716 * *$ & -0.055 & 0.040 \\
\hline PM-27 & $2.317 * *$ & 0.550 & 0.233 & 0.041 & -0.312 & $1.078 * *$ & -0.504 \\
\hline
\end{tabular}

*Significant at $\mathrm{p}=0.05 \%$ level, $* *$ Significant at $\mathrm{p}=0.01 \%$ level 
Table.4.1 Specific combining ability effects of hybrids for different characters

\begin{tabular}{|c|c|c|c|c|c|c|}
\hline & Days to $50 \%$ & Days to & Plant & Number of & Number of & Length of \\
\hline Characters & Flowering & maturity & height & Primary & Secondary & siliqua \\
\hline & & & $(\mathrm{cm})$ & Branches/ plant & Branches /plant & $(\mathrm{cm})$ \\
\hline Laxmi x Pusa Bold & $-2.40 * *$ & 0.30 & $5.22 * *$ & -0.43 & -0.18 & -0.20 \\
\hline Laxmi x Kranti & 0.86 & 0.63 & $7.82 * *$ & $0.63 *$ & -0.11 & 0.24 \\
\hline Laxmi x Varuna & 1.13 & 0.63 & 1.81 & 0.16 & $0.88 * *$ & -0.05 \\
\hline Laxmi x PM - 27 & 0.40 & -1.56 & $-14.85 * *$ & -0.36 & $-0.58 *$ & 0.02 \\
\hline Pusa Agrim x Pusa Bold & 1.18 & 0.13 & $5.09 * *$ & -0.10 & $-0.51 *$ & 0.10 \\
\hline Pusa Agrim x Kranti & 0.11 & 1.46 & $-22.17 * *$ & $-0.70 *$ & -0.45 & $-0.31 *$ \\
\hline Pusa Agrim x Varuna & 0.38 & -1.53 & $7.85 * *$ & 0.50 & $0.55^{*}$ & 0.18 \\
\hline Pusa Agrim x PM -27 & $-1.68 *$ & -0.06 & $9.21 * *$ & 0.30 & 0.41 & 0.02 \\
\hline C.G. Sarson x Pusa Bold & 1.26 & 0.71 & $-11.06^{* *}$ & 0.06 & 0.40 & -0.10 \\
\hline C.G. Sarson x Kranti & $-1.46^{*}$ & -0.28 & $9.07 * *$ & -0.53 & 0.13 & 0.007 \\
\hline C.G. Sarson x Varuna & -1.20 & 0.38 & $-17.06 * *$ & -0.33 & $-0.53 *$ & 0.04 \\
\hline C.G. Sarson x PM - 27 & $1.400^{*}$ & -0.81 & $19.05^{* *}$ & $0.80 * *$ & 0.32 & 0.05 \\
\hline RH - 406 X Pusa Bold & $-1.56^{*}$ & 0.63 & $14.67 * *$ & 0.15 & 0.23 & 0.10 \\
\hline RH - 406 X Kranti & 1.03 & -1.36 & -2.26 & -0.11 & -0.36 & 0.14 \\
\hline RH - 406 X Varuna & 0.30 & -0.36 & $-9.33 * *$ & 0.08 & -0.36 & -0.14 \\
\hline RH - 406 X PM - 27 & 0.23 & 1.10 & $-3.07 *$ & -0.11 & 0.50 & -0.10 \\
\hline DRMR IJ - 31 X Pusa Bold & $1.517 *$ & -1.78 & $-13.92 * *$ & 0.31 & 0.06 & 0.11 \\
\hline DRMR IJ - 31 X Kranti & -0.55 & -0.45 & $7.53 * *$ & $0.71 *$ & $0.80 * *$ & -0.07 \\
\hline DRMR IJ - 31 X Varuna & -0.61 & 0.88 & $16.73 * *$ & -0.41 & $-0.53^{*}$ & -0.03 \\
\hline DRMR IJ - 31 X PM - 27 & -0.35 & 1.35 & $-10.34 * *$ & $-0.61 *$ & -0.33 & 0.003 \\
\hline
\end{tabular}

*Significant at $\mathrm{p}=0.05 \%$ level, **Significant at $\mathrm{p}=0.01 \%$ level 
Table.4.2 Specific combining ability effects of hybrids for different characters

\begin{tabular}{|c|c|c|c|c|c|c|c|}
\hline Characters & $\begin{array}{l}\text { Main } \\
\text { raceme }\end{array}$ & Siliqua on & Seed/siiqua & Weight & Content & Yield/ plant & Index $(\%)$ \\
\hline Laxmi x Pusa Bold & $-2.15^{*}$ & 0.38 & -0.002 & 0.30 & 0.39 & $1.72 *$ & $-2.15^{*}$ \\
\hline Laxmi x Kranti & $3.11 * *$ & $0.71 *$ & -0.17 & $-1.46 * *$ & 0.62 & $-2.04 *$ & $3.11 * *$ \\
\hline Laxmi x Varuna & $5.25 * *$ & -0.28 & 0.17 & $2.41 * *$ & $1.35 *$ & -0.58 & $5.25 * *$ \\
\hline Laxmi x PM - 27 & $-6.21 * *$ & $-0.81 * *$ & 0.008 & $-1.25 *$ & $-2.37 * *$ & 0.90 & $-6.21 * *$ \\
\hline Pusa Agrim x Pusa Bold & $-4.40 * *$ & 0.21 & 0.02 & $-1.33 *$ & -0.86 & 1.03 & $-4.40 * *$ \\
\hline Pusa Agrim x Kranti & -0.46 & -0.45 & 0.05 & $1.57 * *$ & -0.30 & $-1.90 *$ & -0.46 \\
\hline Pusa Agrim x Varuna & $3.00 * *$ & -0.45 & -0.04 & $-1.13^{*}$ & 0.04 & -0.88 & $3.00 * *$ \\
\hline Pusa Agrim x PM -27 & $1.86^{*}$ & $0.68 *$ & -0.03 & 0.89 & $1.13 *$ & $1.75^{*}$ & $1.86^{*}$ \\
\hline C.G. Sarson x Pusa Bold & $2.10^{*}$ & $1.05^{* *}$ & 0.12 & -0.15 & 0.81 & $-2.08 * *$ & $2.10^{*}$ \\
\hline C.G. Sarson x Kranti & -1.30 & $-0.61 *$ & -0.05 & 0.02 & -0.36 & $1.58 *$ & -1.30 \\
\hline C.G. Sarson x Varuna & $-6.50 * *$ & 0.05 & -0.006 & $-1.23 *$ & -0.87 & 0.86 & $-6.50 * *$ \\
\hline C.G. Sarson x PM - 27 & $5.70 * *$ & -0.48 & -0.06 & $1.37 *$ & 0.42 & -0.37 & $5.70 * *$ \\
\hline RH - 406 X Pusa Bold & $3.26 * *$ & $-0.86 * *$ & -0.02 & 0.85 & 0.30 & $-2.02 *$ & $3.26 * *$ \\
\hline RH - 406 X Kranti & $1.86 *$ & $0.80 *$ & 0.09 & -0.28 & -0.39 & $2.25 * *$ & $1.86^{*}$ \\
\hline RH - 406 X Varuna & $-2.66 * *$ & $-0.86 * *$ & -0.02 & -0.62 & -0.14 & 0.85 & $-2.66 * *$ \\
\hline RH - 406 X PM - 27 & $-2.46^{* *}$ & $0.93 * *$ & -0.04 & 0.05 & 0.23 & -1.08 & $-2.46^{* *}$ \\
\hline DRMR IJ - 31 X Pusa Bold & 1.18 & $-0.78 *$ & -0.12 & 0.33 & -0.65 & 1.34 & 1.18 \\
\hline DRMR IJ - 31 X Kranti & $-3.217 * *$ & -0.45 & 0.07 & 0.15 & 0.44 & 0.10 & $-3.217 * *$ \\
\hline DRMR IJ - 31 X Varuna & 0.91 & $1.55 * *$ & -0.09 & 0.57 & -0.37 & -0.24 & 0.91 \\
\hline DRMR IJ - 31 X PM - 27 & 1.11 & -0.31 & 0.13 & $-1.07 *$ & 0.58 & -1.20 & 1.11 \\
\hline
\end{tabular}

$*$ Significant at $\mathrm{p}=0.05 \%$ level, $* *$ Significant at $\mathrm{p}=0.01 \%$ level 
Among the testesr Kranti has negatively significant GCA effect whereas PM - 27 has positive GCA effects were reported. The positive GCA effects indicate their usefulness in breeding for seed yield per plant. Both line and tester were found to have highly significant GCA effect. The lines C.G. Sarson showed negative significant GCA effects where as highly positive GCA effect was observed in Laxmi. Among the testers no significant GCA effect where reported. The positive GCA effects indicate their usefulness in breeding for harvest index. Among lines, positive significant GCA value for Laxmi(1.07) and Pusa Agrime (0.7) for seed yield per plant makes them good general combiners. Among the testers, PM - 27 (1.07) were found to be good combiners for the same trait. The hybrids, C.G. Sarson x Pusa Bold (1.35) showed the highest SCA value followed by C.G. Sarson x Kranti (1.13)for "seed yield per plant."

High PCV (Phenotypic coefficient of variation) and GCV (Genotypic coefficient of variation) were observed in seed yield per plant (g) followed by number of secondary branches per plant. The highest heritability were observed for the character plant height followed by length of main raceme $(\mathrm{cm})$ and seed yield per plant (g). High genetic advance as percent of mean was reported for the seed yield per plant $(\mathrm{g}$ ) followed by length of main raceme $(\mathrm{cm})$. Higher PCV coupled with high GCV, heritability and genetic advance as percent of mean were reported character seed yield per plant (g). Days to 50\% flowering showed positive correlation with primary branches per plant, length of siliqua, length of main raceme and harvest index. The maximum direct effect towards seed yield $(\mathrm{g})$ were reported in number of secondary branches per plant followed by number of seed per siliqua, number of primary branches per plant, test weight and plant height. Parent Laxmi is found to be the best general combiner followed by Varuna, RH - 406, C.G. Sarson, PM - 27 and Pusa Bold where as the best specific combiner among the twenty hybrids are C.G.Sarson x Pusa Bold and Pusa Agrime $\mathrm{x}$ Varuna for different characters. Among the hybrids C.G.Sarson x Kranti and Laxmi $x$ Varuna were reported to be the best for heterosis effect for all characters taken under study.

\section{References}

Anonymous, 2016. Government of India. States Paper on Oilseeds.

Kempthorne, O. 1957. An introduction to Genetic Statistics. John Wiley and Sons Inc., New York.

Niranjana, M., Akabari, V.R., Sasidharan, N., and Jadeja, G.C. 2014. Diallel analysis for yield and its contributing characters in Indian mustard" [Brassica juncea L.) Czern \& Coss]. Elec J. Plant. Bree. 5(2): 197-202.

Yadava, D.K. Singh, N., Vasudev, S., Singh, R., Singh, S., Giri, S.C., Dwivedi, V.K. and Prabhu K.V. 2012. Combining ability and heterobeltiosis for yield and yield-contributing traits in Indian mustard (Brassica juncea). Ind. J. Agricultural Sci. 82 (7): 563-570.

\section{How to cite this article:}

Ashish Sahu, J. L. Salam, Surbhi Verma and Stanley Samuel. 2020. Combining Ability and Heterosis for Seed Yield and its Attributing Traits in Indian Mustard (Brassica juncea L. Czern \& Coss). Int.J.Curr.Microbiol.App.Sci. 9(07): 720-727.

doi: https://doi.org/10.20546/ijcmas.2020.907.083 